\title{
SAR Evaluation Towards Breast Cancer in Hyperthermia Treatment
}

\author{
Bibi Sarpinah Sheikh Naimullah \\ Faculty of Engineering, Universiti \\ Malaysia Sarawak \\ Faculty of Engineering, Universiti \\ Teknologi MARA, Sarawak \\ bpina@uitm.edu.my \\ Mazlina Mansor Hassan \\ Faculty of Engineering, Universiti \\ Malaysia Sarawak \\ Faculty of Engineering, Universiti \\ Teknologi MARA, Sarawak \\ mazlinamansor@uitm.edu.my
}

\author{
Kasumawati Lias \\ Faculty of Engineering, Universiti \\ Malaysia Sarawak, Sarawak \\ 1kasumawati@unimas.my \\ Hazrul Mohamad Basri \\ Faculty of Engineering, Universiti \\ Malaysia Sarawak, Sarawak \\ mbhazrul@unimas.my
}

\author{
Norlida Buniyamin \\ Faculty of Engineering, Universiti \\ Teknologi MARA, Sarawak \\ nbuniyamin@uitm.edu.my
}

Dzufi Izura Ispawi

Faculty of Engineering, Universiti

Teknologi MARA, Sarawak

dzufiizurai@uitm.edu.my

\begin{abstract}
Hyperthermia treatment is an alternative treatment that can be considered in breast cancer. This treatment uses Electromagnetic waves as a source of heat to destroy cell cancer. The main challenge for this treatment is the focusing capability and the existence of a wide area of unwanted hotspots at the surrounding healthy cell. Therefore, in this paper, the desired penetration depth and focus position distance are obtained and compared with different frequencies and substrates. The desired penetration depth and focus position distance are basically determined from the statistical analysis of real mammogram breast cancer images. The simulation with SEMCAD $X$ is then conducted to select the best operating frequency and substrate to achieve the great penetration depth and focus position distance on the treated tissue. Based on the results, the different operating frequencies with different substrates have provided different penetration depth and focus position distance. The unwanted hotspot areas are also different. In conjunction with that, further research needs to be carried out in order to obtain the best operating frequency with the most suitable substrate to be used that may attain the required results on the treated tissue, mainly when different cancer depths and focus position distances are concerned.
\end{abstract}

Keywords-hyperthermia, penetration depth, focus position distance, hot spot, SAR

\section{INTRODUCTION}

Hyperthermia utilizes high heat that applies temperature in the range of $42^{\circ} \mathrm{C}$ to $45^{\circ} \mathrm{C}$ at a certain duration of time. The purpose is to ensure the denaturation of the cancer cells into necrotic tissue with minimal damage to surrounding healthy tissue[1][2]. Based on previous studies, the main significant problem that contributed to the low success of hyperthermia treatment was it poor focus position distance, with wider unwanted hot spots, which may affect the surrounding healthy tissue [3][4][5][6][7][8][9][10][11][12], and then leads to adverse health effects. The problems became more massive when non-invasive treatment was concerned, especially for those hyperthermia procedures with the external hyperthermia applicator and electromagnetics heating medium.

Furthermore, in [13][14][15][16][17][18] the focus position distance was small in diameter and focused. However, it may not be sufficient for this research condition environment as different treated areas may contribute to different outcomes since each treated tissue has different electrical properties that may affect the absorption rate of heat on the treated tissues. Meanwhile, in[19][20], the focus area was wider and contributed to unwanted hot spots on the surrounding healthy area. The focus position should cover the treated area in the range of $+5 \mathrm{~mm}$ in order to ensure the treated area has been heated sufficiently.

In this research, it mainly aims to improve focusing capability as well as to reduce unwanted hotspots in order to enhance the effectiveness of hyperthermia procedure for breast cancer treatment.

Hyperthermia treatment procedure (HTP) requires a sufficient amount of heat. The heat source for HTP is from Electromagnetic waves (EM), while the EM heating generated an electric field. Change in the electric field creates magnetically, and vice versa produces propagation wave. The wave propagated and penetrated the skin tissue[21]. The long EM waves have low frequencies and carried less energy if compared to high frequency. The absorption into the skin tissue is determined by the Specific Absorption Rate (SAR)[22]. It is the parameter that uses to observe the heat distribution and radiation absorption in human tissue, in this case, is the cancer cells.

The common used operating frequency for HTP is $434 \mathrm{MHz}, 915 \mathrm{MHz}$, and $2450 \mathrm{MHz}$. These frequencies are based on Industry, Scientific, and Medical (ISM) frequency range[23]. Based on previous studies, different operating frequencies, different focus position distances, and sizes of heat radiation are obtained [24].

The scope of this research is to determine the best operating frequency and substrate to obtain the desired penetration depth and focus position distance. The desired penetration depth and focus position distance extract from the statistical analysis mammogram image. The simulation experiment outcomes need to satisfy the desired penetration depth and focus position distance. The following section discusses the research methodology. 